\title{
La culture de l'éducation de la petite enfance en Italie
}

Early childhood education culture in Italy

La cultura de la educación de la pequeña infancia en Italia

Maria Cristina Picchio et Tullia Musatti

\section{OpenEdition}

\section{Journals}

Édition électronique

URL : http://journals.openedition.org/ries/888

DOI : $10.4000 /$ ries.888

ISSN : 2261-4265

Éditeur

Centre international d'études pédagogiques

Édition imprimée

Date de publication : 1 avril 2010

Pagination : 101-108

ISSN : 1254-4590

Référence électronique

Maria Cristina Picchio et Tullia Musatti, « La culture de l'éducation de la petite enfance en Italie », Revue internationale d'éducation de Sèvres [En ligne], 53 | avril 2010, mis en ligne le 01 avril 2013, consulté le 10 décembre 2020. URL : http://journals.openedition.org/ries/888 ; DOI : https://doi.org/ $10.4000 /$ ries.888 


\section{La culture de l'éducation de la petite enfance en Italie}

\section{Mariacristina Picchio Tullia Musatti}

Autour des institutions éducatives pour la petite enfance, en Italie, s'est développée une culture de l'enfance en relation avec le développement normal des jeunes enfants dans un contexte éducatif, les besoins de soutien social et matériel de leurs parents et les réponses que les politiques locales et nationales peuvent offrir à ces besoins. Notre contribution présente les éléments historiques et institutionnels qui ont permis le développement de cette culture, ainsi que les aspects qui la caractérisent.

Nous voulons montrer que la culture de l'enfance en Italie, tout en profitant de l'apport essentiel de la recherche scientifique et de la filiation d'approches pédagogiques importantes, s'est développée à l'intérieur et autour des institutions pour la petite enfance. Elle s'est donc profondément nourrie des expériences culturelles et politiques des territoires dans lesquels ces institutions éducatives ont été créées.

\section{LES MUNICIPALITÉS : \\ DES PARTIES PRENANTES}

Dans l'Italie d'après la Deuxième Guerre mondiale, le rôle des communautés locales dans l'éducation préscolaire a été essentiel pour remplacer et solliciter l'intervention de l'État qui n'envisageait pas un engagement important dans les services pour la petite enfance. Les premières expériences d'école pour les enfants de 3 à 5 ans, réalisées par des collectifs de femmes bientôt relayés par certaines communes d'Italie du Centre et du Nord, précédèrent la création d'une école maternelle publique d'État (1968). Bien qu'aujourd'hui la scuola dell'infanzia publique d'État accueille $60 \%$ des enfants d'âge préscolaire, les écoles communales demeurent importantes dans quelques grandes villes ou territoires, à côté des écoles maternelles privées, religieuses et laïques.

Par la suite, le ministère de l'Éducation a produit des documents d'orientation pédagogique. Bien que ces documents aient été adressés aux écoles maternelles publiques d'État, ils ont constitué un cadre de référence théorique pour les écoles municipales aussi. Toutefois, il faut remarquer que, dans ces documents, on retrouve des traces de la culture pédagogique qui s'était entre-temps formée dans les scuole dell'infanzia communales de villes comme Reggio Emilia, Modène, Pistoia, Turin, Milan, ou Gênes. 
Les institutions éducatives pour les enfants de moins de 3 ans ont été intégrées de manière naturelle dans ce parcours culturel, et ceci dans plusieurs villes. En fait, les nidi, comme les crèches françaises, accueillent les enfants âgés de moins de 3 ans et ont été institués comme services publics par une loi d'État de 1971, mais leur programmation, leur réglementation et leur gestion ont été confiées aux administrations municipales et régionales. Depuis leurs débuts, ces institutions éducatives relèvent donc de l'intervention des administrations locales : cette dépendance a eu des conséquences importantes dans la détermination de leur profil culturel (Mantovani, 2007).

L'absence d'une gouvernance centralisée des crèches a eu un impact déterminant, dont le plus remarquable est la grande disparité sur le territoire national, tant pour l'offre quantitative que qualitative. Bien que la couverture nationale des places par rapport au nombre d'enfants de moins de 3 ans se situe autour de $10 \%$, les données sont très variables selon les régions et les villes, allant d'1 \% à 30-40\%. En outre, dans les villes où l'investissement local dans le secteur a été important, on a assisté non seulement à une extension quantitative des nidi, mais aussi à la réalisation d'expériences d'excellence reconnues au niveau international et à la création d'institutions d'un nouveau type, tels que les lieux d'accueil parents-enfants.

\section{UN INVESTISSEMENT SUR LA QUALITÉ PROFESSIONNELLE DU PERSONNEL}

Ces expériences d'excellence ont pu être réalisées essentiellement grâce aux investissements des communautés locales en matiière de qualité professionnelle du personnel tant dans les nidi que dans les scuole dell'infanzia. Les administrations locales ont donné une grande importance à la détermination du ratio enfants/adulte aussi bien qu'à l'organisation du roulement du personnel, afin de garantir au mieux la présence de plusieurs éducateurs dans les plages horaires les plus importantes de la journée éducative. Il faut souligner aussi que le contrat national de travail du personnel dans les institutions éducatives municipales prévoit un montant annuel d'heures pour la planification du travail éducatif en équipe et la formation professionnelle. Ces heures ont été utilisées par les administrations locales pour promouvoir et soutenir la qualité de l'offre éducative des institutions pour la petite enfance, en garantissant aux personnels des parcours de formation continue.

Ce contexte a constitué, dans plusieurs localités, un terrain formidable pour développer une réflexion pédagogique originale et mener des expériences éducatives innovantes. Il faut dire aussi que, puisque pendant longtemps, seule une formation de niveau bac (bac socio-pédagogique) était requise pour les personnels, la carence de savoirs codifiés a paradoxalement contribué à motiver cette réflexion et à transformer les occasions de formation continue en laboratoires d'innovation éducative. 
Ceci a été vrai, en particulier, pour les personnels des nidi qui arrivaient à la rencontre avec les tout-petits et leurs familles sans aucun bagage de connaissances spécifiques, et en reconnaissant que les expériences réalisées dans les scuole dell'infanzia ne pouvaient être reproduites pour les enfants plus jeunes sans modifications substantielles. Les parcours de formation continue offraient donc l'occasion de repenser intégralement la signification et les modalités de l'action éducative pour les tout petits.

\section{LA RENCONTRE ENTRE PRATIQUE ÉDUCATIVE ET RECHERCHE SCIENTIFIQUE}

Dans ce processus d'élaboration de la réflexion éducative sur la petite enfance, une contribution très importante a été donnée par une nouvelle génération de chercheurs et d'universitaires, qui au début des années quatre-vingts portaient un nouvel regard sur le développement du jeune enfant, se référant à la pensée de Piaget et Wallon, aux travaux d'Irène Lézine, Mira Stambak et d'autres chercheurs du CRESAS, mais aussi aux approches cognitivistes et de la psycholinguistique américaine. Leur intérêt pour les performances communicatives, sociales et cognitives des enfants au cours des premières années les a amenés à s'intéresser aux nidi afin d'y trouver des sujets d'étude. En outre, dans ces mêmes années, les études éthologiques permettaient de voir dans le nido l'occasion d'étudier les processus de socialisation dans un contexte naturel. Les recherches menées ont ainsi contribué à définir une nouvelle image de l'enfant et de ses compétences sociales, affectives et cognitives dans la société, et à diffuser une nouvelle image des institutions éducatives pour la petite enfance, en tant que lieu de vie quotidienne normale pour les tout-petits (Musatti, 2007).

La rencontre entre les centres d'intérêt de la recherche scientifique et les préoccupations éducatives a été particulièrement féconde. Tout en offrant aux chercheurs un observatoire exceptionnel de la petite enfance, elle suggérait au personnel des nidi d'observer et d'interpréter les conduites et les besoins des enfants ainsi que de réfléchir aux modalités adaptées pour y répondre.

Une première conséquence importante de cette rencontre a été le fait que la culture de l'enfance en Italie ne se présente actuellement pas comme un corps de connaissances et de savoirs systématiques produits par des institutions académiques, puisqu'elle s'est développée dans une forte synergie entre le monde des institutions éducatives et le monde de la recherche. Deuxièmement, le personnel a souvent joué un rôle de partie prenante dans les recherches conduites à l'intérieur et autour des institutions éducatives pour la petite enfance, puisque la plupart des études ont été réalisées dans le cadre de formations continues et de parcours de recherche-action. C'est ainsi qu'ont été explorés les processus d'apprentissage et de socialisation des petits enfants, les dynamiques relationnelles entre adultes et enfants, la qualité de l'offre éducative, et que se sont développés des pratiques 
innovantes. Actuellement, il existe un consensus sur le fait que les formations continues ne doivent pas viser à transmettre des contenus disciplinaires ou des stratégies didactiques, mais plutôt à offrir aux personnels l'occasion de construire des savoirs autour des soins et de l'éducation de l'enfance, à partir de l'observation et de l'analyse de pratiques éducatives réalisées dans les institutions.

Une autre impulsion importante est venue de la présence et des initiatives du Gruppo Nazionale Nidi Infanzia, une association fondée en 1980 par Loris Malaguzzi qui, de façon originale, a réuni personnels des institutions éducatives, chercheurs et universitaires, administrateurs locaux et gestionnaires, sur la base d'un intérêt culturel, politique et professionnel commun autour de la petite enfance. L'association a ainsi contribué à la construction et à la diffusion de la culture de l'enfance en Italie et à la continuité de son renouvellement. Elle a offert des stimulations culturelles, provenant d'expériences éducatives et d'environnements culturels et scientifiques variés et multidisciplinaires.

\section{ÉDUCATEURS ET COORDINATEURS}

Les expériences de gestion des scuole per l'infanzia réalisées par les administrations municipales ont aussi eu une grande influence sur l'encadrement du personnel des nidi. Tous les personnels, presque exclusivement des femmes, prenant soin des enfants dans les nidi et dans les autres institutions pour la petite enfance, ont été globalement appelés éducatrices et considérés comme telles. Le profil du coordinateur psychopédagogique et la fonction de coordination mis en place initialement pour les écoles ont été étendus à tout le système des services municipaux accueillant les enfants de 0 à 6 ans.

$\mathrm{Au}$ cours de ces dernières années, cette fonction s'est répandue et aujourd'hui, presque toutes les communes qui gèrent des institutions éducatives ont embauché un nombre suffisant de coordinateurs. Ces coordinateurs, à la différence de leurs homologues dans les villes françaises (Baudelot, Rayna, Mayer et Musatti, 2003), ont la responsabilité d'un nombre variable, mais néanmoins réduit, d'institutions éducatives pour la petite enfance (dans lesquelles il n'y a pas de directrice). Ils ont pour mission de soutenir et d'accompagner les pratiques éducatives à l'intérieur de chaque institution et de construire et promouvoir des liens en réseau entre les différents services présents sur un même territoire. Ils sont organisés en équipes municipales de coordination, proposent des projets et développent des politiques locales destinées aux enfants et à leurs familles.

La fonction des coordinateurs s'est révélée fondamentale pour promouvoir et soutenir la qualité de l'éducation, en accompagnant les pratiques de chaque institution et le travail en réseau entre toutes les institutions éducatives de la ville.

Nous avons présenté jusqu'ici les éléments culturels et d'organisation qui, selon nous, ont particulièrement influencé certaines pratiques éducatives des institutions pour la petite enfance en Italie. Naturellement, ces éléments se 
sont développés différemment selon les réalités locales, et ont donné lieu à des pratiques variées en fonction de l'attention spécifique portée par les responsables et les personnels aux institutions éducatives pour la petite enfance, ainsi que des choix politiques et culturels inspirés par les particularités de chaque contexte.

\section{L'ATTENTION AUX RAPPORTS ENTRE L'INSTITUTION ET LA FAMILLE}

Depuis leur création, les institutions éducatives municipales pour la petite enfance ont été les premières à laisser un espace aux parents, en leur donnant un rôle de protagonistes pour participer à la gestion et en les considérant non seulement des utilisateurs/clients, mais comme des sujets et des interlocuteurs actifs.

L'attention portée aux rapports avec les familles a induit une réflexion approfondie sur la signification de la première entrée de l'enfant et de ses parents dans l'institution éducative, sur les stratégies d'accueil et sur l'accompagnement de la phase de séparation entre l'enfant et ses parents (Mantovani, Restuccia Saitta et Bove, 2000).

De multiples stratégies visant à promouvoir la participation des parents à la vie des nidi et des scuole dell'infanzia ont été élaborées et mises en ouvre autour de la communication avec les parents, sur le thème du développement des enfants, conduisant à des pratiques de plus en plus calibrées, révisées en fonction de la pluralité et de la spécificité des demandes et des besoins des parents (Catarsi, 2008). Cette écoute de la voix des familles a permis de repérer la demande naissante des parents de trouver, dans les institutions éducatives pour la petite enfance, un soutien professionnel à leur expérience de parents, aussi bien que des espaces pour socialiser et échanger entre parents difficultés, doutes et points de vue sur l'éducation. Les familles ont ainsi pu sortir de l'isolement matériel et psychologique qui caractérise de plus en plus souvent leur condition actuelle, quand elles ont de très jeunes enfants. La reconnaissance de ces besoins a conduit à la création de nouveaux services, comme les Centres pour parents et enfants, et à repenser le rôle des institutions éducatives pour la petite enfance dans la société moderne : non seulement des lieux éducatifs pour les plus petits, mais des lieux de soutien pour les parents et des lieux de rencontre sociale pour les familles (Musatti et Picchio, 2005).

L'Italie ayant vu augmenter rapidement, ces dernières années, le nombre de travailleurs immigrés, on trouve aujourd'hui, dans les nidi et les scuole dell'infanzia, un nombre important d'enfants nés de parents étrangers. Par conséquent, les institutions éducatives pour la petite enfance ont dû repenser leurs pratiques des relations entre institutions éducatives et familles dans une perspective multiculturelle (Favaro, Mantovani et Musatti, 2008). 


\section{L'ATTENTION À LA QUALITÉ DU CONTEXTE}

L'observation et la valorisation des dynamiques sociales et cognitives dans les institutions a permis d'offrir aux enfants accueillis dans les nidi et dans les scuole dell'infanzia un contexte de qualité et la possibilité de faire des expériences de qualité, plutôt que des parcours d'apprentissage prétablis. Dans une institution éducative, l'enfant est exposé à une situation sociale traversée de relations interpersonnelles complexes, avec la présence d'une pluralité de sujets qui, par leur présence, par leurs actions, par leurs modalités d'interaction, contribuent à définir la qualité du contexte dont l'enfant fait l'expérience.

Dans la culture italienne des institutions éducatives pour la petite enfance, on accorde une grande attention à la promotion de dynamiques sociales positives dans le groupe d'enfants et d'adultes et à la construction de relations stables entre enfants ainsi qu'entre enfants et adultes. C'est justement pour souligner l'importance de la dimension sociale et relationnelle dans la qualité du contexte proposé par les institutions éducatives pour les plus petits que la pédagogie du nido a été définie comme pédagogie des relations (Bondioli et Mantovani, 1987). Dans cette pédagogie, on considère que le développement cognitif de l'enfant doit être soutenu par un contexte de vie et d'expérience riche et varié, mais en même temps stable, permettant la continuité d'un parcours cognitif significatif. Ainsi, la proposition d'activités spécifiques de jeu et d'exploration a-t-elle du sens si elle est quotidienne et non pas ponctuelle. Il s'agit de soutenir et promouvoir le développement des connaissances des enfants, tout en refusant de solliciter des parcours d'apprentissage de type scolaire.

Par ailleurs, on considère que la qualité de l'expérience des enfants au sein d'une institution éducative est définie par des aspects structurels et organisationnels, par exemple les petits groupes d'enfants et la présence des éducateurs au sein des groupes. Une grande importance a été donnée à l'organisation du temps et de l'espace dans l'institution éducative. Le fait que la structuration et l'articulation des espaces (Galardini, 2003 ; Rinaldi, 2001), tout comme l'organisation des temps dans la journée, ont une influence significative sur les rencontres sociales entre enfants est un postulat largement partagé. Cette influence porte sur la qualité de l'interaction communicative et relationnelle ainsi que sur les parcours de connaissance que les enfants développent, en termes de contenus et de complexité.

\section{Collégialité ET DOCUMENTATION}

La culture de l'enfance a trouvé un contexte important de développement dans les occasions de travail en équipe : en effet, la possibilité d'heures de travail rétribuées hors de la présence des enfants permet la discussion des pratiques éducatives entre les différents personnels concernés (éducateurs, coordinateurs, personnel auxiliaire). Dans les institutions italiennes pour la petite 
enfance, la collégialité est l'espace qui permet l'élaboration de projets pédagogiques, leur réalisation, leur évaluation.

Dans les contextes éducatifs pour jeunes enfants, la discussion et la coopération entre les adultes qui en prennent soin représente non seulement un choix, mais une nécessité. En effet, la qualité de l'expérience vécue par un enfant est fortement influencée par la cohérence des interventions des adultes et par la complémentarité des propositions que ces derniers lui adressent. Cette dimension de la collégialité est donc une composante essentielle du travail du personnel dans ces institutions, et le partage des réflexions sur les pratiques et sur leurs effets au niveau de l'expérience des enfants est la base sur laquelle cette collégialité se réalise.

La collégialité est une condition du travail du personnel des institutions éducatives pour la petite enfance, l'objectif n'étant jamais atteint définitivement : construire des idées communes sur les soins et l'éducation de l'enfance et sur le rapport avec les familles est le résultat d'un processus continu, au cours duquel les points de vue des différents personnes doivent s'exprimer dans un travail de confrontation et de négociation permanent et dans le but commun de mieux comprendre l'expérience des enfants et d'améliorer les pratiques éducatives. En ce sens, l'échange et la discussion entre personnels est non seulement une pratique professionnelle, mais également une pratique démocratique.

En Italie, documenter les parcours d'expérience des enfants est une pratique professionnelle enracinée dans les institutions éducatives pour la petite enfance. C'est dans la ville de Reggio Emilia que cette documentation a été particulièrement approfondie (Rinaldi, 2005), ce qui a contribué à la diffuser dans nombre d'autres villes. À travers la documentation, les capacités d'écoute et de compréhension des réflexions des enfants s'affinent. C'est aussi à travers la documentation que l'on met à la disposition des familles et de toute la communauté locale le patrimoine d'expériences et de connaissances capitalisé à l'intérieur des nidi et des scuole per l'infanzia.

Nous voulons aussi souligner la valeur particulière des pratiques de documentation pour soutenir et promouvoir l'exercice de la collégialité. La documentation, en effet, se révèle un instrument important dans la construction d'une mémoire partagée de l'expérience faite avec les enfants dans une équipe éducative et dans l'élaboration, à partir de cette expérience, de nouveaux savoirs partagés, à travers l'exercice de la réflexivité. 


\section{BIBLIOGRAPHIE}

BAUDELOT O., RAYNA S., MAYER S., MUSATTI T. (2003) : «A comparative analysis of the function of coordination of Early Childhood Education and Care in France and Italy ». Early Years Education, 11, 105-116.

BONDIOLI A., MANTOVANI S. (a cura di) (1987) : Manuale critico dell'Asilo Nido. Milano: Franco Angeli.

CATARSI E. (2008) : Pedagogia della famiglia. Roma: Carocci.

FAVARO G., MANTOVANI S., MUSATTI T. (coord.) (2008) : Une crèche pour apprendre à vivre ensemble. Ramonville Saint-Agne (FR) : Éditions Eres.

GALARDINI A.L. (a cura di) (2003) : Crescere al nido. Roma: Carocci.

MANTOVANI S. (2007) : "Early Childhood Education in Italy ». In New R. S., Cochran M., Early Childhood Education. An International Encyclopedia. Vol. 4 (pp. 1110-1115). Westport (CT): Praeger Publishers.

MANTOVANI S., RESTUCCIA SAITTA L., BOVE C. (2000) : Attaccamento e inserimento. Stili e storie di relazioni al nido. Milano: Franco Angeli.

MUSATTI T. (2007) : «La signification des lieux d'accueil pour la petite enfance aujourd'hui ». In Brougère G. et Vandenbroeck M. (coord.), Repenser l'éducation des jeunes enfants (pp. 209-226). Bruxelles : Peter Lang.

MUSATTI T., PICCHIO M. (2005) : Un luogo per bambini e genitori nella città. Trasformazioni sociali e innovazioni nei servizi per l'infanzia e le famiglie. Bologna: Il Mulino.

RINALDI C. (2001) : In Gandini L., Pope Edwards C. (Eds.), Bambini. The Italian Approach to Infant/Toddler Care. (pp. 49-54). New York (NY): Teachers Collage Press. RINALDI C. (2005) : Dialogue with Reggio Emilia. London: Routledge. 